\title{
Evaluación agroindustrial de tres variedades pre comerciales de trigo
}

Marlene Vázquez Mendoza ${ }^{1}$

\section{Resumen}

El propósito de evaluar 3 variedades pre comercial de trigo BS16, Norteña y Josecha, consiste en identificar a través de los análisis, las propiedades físicas y mecánicas, análisis proximal, reológicos y evaluación sensorial empleando métodos físicos como peso hectolítrico, estimación tanto de la calidad física del grano, como de la calidad molinera, método de dureza molienda en molino experimental Bulher automático para la obtención de harina fina, análisis proximal empleando el método de Goldfisch para la determinación de extracto etéreo, método de digestión acida y alcalina para la determinación de fibra, método Kjeldhal para nitrógeno, método Farinógrafo de Brabender utilizando un equipo Micro-Farinógrafo Brabender Mod-No. 8 110, determinación de propiedades dinámicas de la masa utilizando el alveógrafo de Chopin donde la masa se extiende bidimensionalmente formando un alveolo, por efecto de la fuerza debida a la presión del aire que se insufla por debajo de una lámina de masa obtenida en condiciones normalizadas, método 10-10 AACC 1962 para la evaluación de panificación y sensorial que determinaron el potencial de las variedades de trigo para su aprovechamiento industrial. Los materiales antes mencionados fueron cultivados en la región de Mixquiahuala de Juárez Hidalgo, obteniendo que la variedad óptima es BS16, que cumple con los parámetros para su industrialización. Los análisis para determinar la calidad panadera de la harina de trigo se realizaron en el Instituto Nacional de Investigaciones Forestales, Agrícolas y Pecuarias (INIFAP) de la zona centro sur (CEVAMEX).

Palabras Clave: Trigo, variedades de trigo, calidad panadera. 


\begin{abstract}
The purpose of evaluating three pre-commercial wheat varieties -BS16, Norteña y Josecha- is identified the physical and mechanical properties, proximate analysis, rheological and sensory evaluation using physical methods such as hectolitre weight, grain physical quality estimation, milling quality, hardness determination at automatic Bulher experimental mill for obtaining fine flour, proximate analysis: ether extract determination by Goldfisch method, acid and alkaline digestion to fibre determination Kjeldahl method for nitrogen quantification, Brabender Farinograph using a Brabender Micro-Farinograph Mod-No . 8110. Calculation of dough dynamic properties using Chopin alveograph where dough is dimensionally extended forming a alveolus due to the force of air pressure blown under a dough sheet obtained under standard conditions. The 10-10 AACC 1962 method was applied for sensory evaluation and baking, determining the potential of wheat varieties for industrial use. The above materials were grown in Hidalgo Juarez Mixquiahuala, and BS16 variety is the optimal one, since it meets industrialization parameters. The analysis to determine wheat flour baking quality was performed at the National Institute for Forestry, Agriculture and Livestock (INIFAP) south central area (CEVAMEX).
\end{abstract}

Keywords: Wheat, wheat varieties, bread quality. 


\section{Introducción}

El campo experimental del Instituto Tecnológico Superior del Occidente del Estado de Hidalgo, cuenta con un área destinada a la producción de cultivos como alfalfa, maíz, cebada, sorgo y trigo. El trigo es un grano que se ha cultivado en este campo experimental en donde las variedades producidas fueron acopladas bajo condiciones de cultivo a cielo abierto, irrigación con aguas negras y suelos mínimamente fertilizados con el objetivo de obtener un grano de calidad apto para su industrialización, mismo que fue analizado minuciosamente aplicando las técnicas químicas y físicas específicas, evaluación de propiedades mecánicas y dinámicas del grano y se determinó la calidad en la harina para industrialización en cada variedad.

Por esta razón una de las instituciones de apoyo fundamental en la realización de esta investigación fue el INIFAP-CEVAMEX, ya que cuenta con las instalaciones y laboratorios con equipos especializados para la aplicación de las técnicas que permiten identificar y caracterizar la harina de trigo para su industrialización.

La fabricación de harina a partir del trigo requiere de un proceso complejo en el cual intervienen muchas etapas, de molienda y tamizado. La eficiencia de la molienda del trigo depende de la efectividad del acondicionamiento o templado del trigo y también del flujo adecuado de materiales a través del molino. Cuando se muele trigo que se ha acondicionado de forma adecuada, el grano endurecido y el germen se desprenden en trozos relativamente grandes y las partículas molidas de endospermo pueden separarse con facilidad de ellos. En general el $72 \%$ del grano de trigo se recupera como harina y el $28 \%$ restante como subproductos (Desrosier, 1999). La harina contiene entre un 65 y un $70 \%$ de almidones, pero su valor nutritivo fundamental está en su contenido, ya que tiene del 9 al 14\% de proteínas; siendo las más importantes la gliadina y la glutenina, además de contener otros componentes como celulosa, grasas y azúcar. (Desrosier, 1999).

\section{Materiales y métodos}

En los meses de mayo - junio de 2012, se realizaron los primeros análisis proximales y en el mes de julio las propiedades físicas y mecánicas, calidad panadera y sensorial. Los cuadros No.1 y No.2 muestran el listado de materiales y equipos que se utilizaron en la realización de los análisis, acondicionamiento del trigo, las propiedades físicas y mecánicas, bromatológicos, reológicos y la evaluación sensorial, respectivamente. 


\section{Cuadro No.1 Listado de materiales que se utilizaron en la realización de los Análisis Fisicoquímicos y Bromatológicos}

\begin{tabular}{|l|l|l|}
\hline No. & Materiales & Cantidad/volumen \\
\hline 1. & Vaso de precipitado & 100,250 y $1000 \mathrm{ml}$ \\
\hline 2. & Pipeta graduada & 5 y $10 \mathrm{ml}$ \\
\hline 3. & Probeta graduada & 50,250 y $1000 \mathrm{ml}$ \\
\hline 4. & Vasos de Berzelius y vasos de Borde Esmerilado & $600 \mathrm{ml}$ y $200 \mathrm{ml}$ \\
\hline 5. & Tubos recolectores & 6 piezas \\
\hline 6. & Agitador de vidrio, porta dedales, anillos de sujeción y espátulas & Un lote \\
\hline 7. & Crisol y cápsula de porcelana & 9 \\
\hline 8. & Pinzas para crisol & 2 \\
\hline 9. & Mechero bunsen & 4 \\
\hline 10. & Desecador & 3 \\
\hline 11. & Tripie, triángulos de porcelana y lámina de asbesto. & 4 \\
\hline 12. & Extensión y eliminador de corriente eléctrica & 2 \\
\hline
\end{tabular}

\section{Cuadro No.2 Listado de equipos que se utilizaron en la realización de los análisis fisicoquímicos, bromatológicos y reológicos.}

\begin{tabular}{|c|c|c|}
\hline No. & Equipos & Características generales \\
\hline 1. & Balanza analítica: & $\begin{array}{l}\text { Marca Ohaous, con precisión en la lectura de sus pesos } \\
\text { realizados con sensibilidad de } 0,1 \mathrm{mg} \text {. }\end{array}$ \\
\hline 2. & Mufla & $\begin{array}{l}\text { Se alcanzan elevadas temperaturas de } 500^{\circ} \mathrm{C} \text { hasta de } \\
1100^{\circ} \mathrm{C} \text {. }\end{array}$ \\
\hline 3. & $\begin{array}{l}\text { Aparato Digestor-dispositi- } \\
\text { vo de destilación Kjeldhal }\end{array}$ & $\begin{array}{l}\text { Es un método para determinar el contenido de proteína } \\
\text { y nitrógeno de substancias orgánicas e inorgánicas, usa } \\
\text { frascos KNA de } 300 \text { ml y pueden manejar tamaños de } \\
\text { muestras desde } 0.5 \text { hasta aproximadamente } 5.0 \text { gramos. }\end{array}$ \\
\hline 4. & $\begin{array}{l}\text { Aparato de extracción de } \\
\text { extracto etéreo Goldfish }\end{array}$ & Aparato de extracción de extracto etéreo Goldfish \\
\hline 5. & $\begin{array}{l}\text { Aparato de digestión de } \\
\text { fibra }\end{array}$ & $\begin{array}{l}\text { Se utiliza en la determinación de fibra cruda, alimentos y } \\
\text { otros productos agrícolas. }\end{array}$ \\
\hline 6. & Molino de disco de piedra. & $\begin{array}{l}\text { Este equipo se utiliza para conocer la dureza del trigo, para } \\
600 \text { gramos. }\end{array}$ \\
\hline 7. & $\begin{array}{l}\text { Balanza Winchester Bus- } \\
\text { hel Meter u Oahu's }\end{array}$ & $\begin{array}{l}\text { Se utiliza para conocer el peso hectolítrico de una muestra } \\
\text { de trigo. }\end{array}$ \\
\hline 8. & $\begin{array}{l}\text { Molino Experimental Bul- } \\
\text { her Automático }\end{array}$ & $\begin{array}{l}\text { Se utiliza para moler trigos duros, ( } 800 \text { y } 500 \text { gramos). } \\
\text { Modelo MLU202.Molino de Rodillo Liso. }\end{array}$ \\
\hline 9. & Alveógrafo de Chopin & $\begin{array}{l}\text { Aparato Reológico para conocer los parámetros más impor- } \\
\text { tantes de la calidad panadera de un trigo. }\end{array}$ \\
\hline 10. & Farinógrafo de Brabender & $\begin{array}{l}\text { Aparato reológico que mide la absorción de agua y compor- } \\
\text { tamiento del amasado. }\end{array}$ \\
\hline
\end{tabular}


El muestreo se llevó a cabo a partir de sacos de trigo que fueron tomados al azar de los cuales se obtuvieron 2 kilogramos de grano de trigo de cada variedad pre comercial; Josecha, BS16 y Norteña.

\section{Acondicionamiento del trigo}

\section{Análisis Físico}

Esta etapa se llevo a cabo en el Instituto Nacional de Investigaciones Forestales, Agrícolas y Pecuarias (INIFAP), se analizan 3 variedades de trigo, mediante muestras representativas en las que se obtuvieron resultados en cada una de los métodos físicos que a continuación se describen.

\section{Limpieza del grano}

En esta etapa se retira la mayor parte de las impurezas del grano, presentes en el mismo como son: Todo lo que no sea trigo, trigo dañado, trigo helado, trigo oreado, trigo germinado, trigo chupado, trigo verde o inmaduro. Imagen No.1 Impurezas presentes en las diferentes variedades a evaluar.

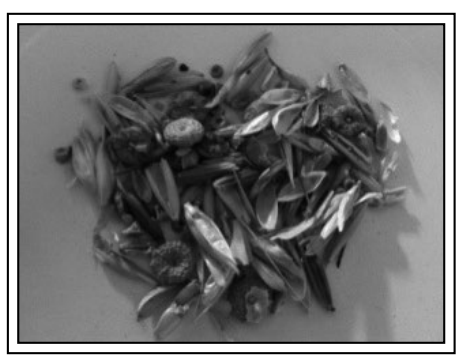

Imagen No.1 Impurezas presentes en las diferentes variedades a evaluar.

Peso Hectolítrico: Es importante porque cuanto más san

o sea (menor cantidad de impurezas, granos dañados o quebrados, picados, o con presencia de cualquier enfermedad), mayor será la proporción de almidón en el grano y mejor será la separación del endospermo del resto del grano. Por lo tanto, cuanto más sano, mayor extracción de harina. A su vez, es una medida de la homogeneidad de la partida de trigo, factor clave en el proceso industrial. Por consiguiente, el peso hectolítrico es una buena estimación tanto de la calidad física del grano, como de la calidad molinera.

Dureza: La dureza del grano, es el método que define que tan duro o blando es el endospermo de la variedad de grano analizado. Por lo que se manifiesta la resistencia que opone al ser fracturado o a la energía requerida para reducir significativamente en la molienda el endospermo a harina o semolina.

\section{Procedimiento}

Paso 1: Acondicionamiento del grano en esta etapa se separa el grano de la impureza que pueda presentar, como lo es retirar materia extraña que en su 
momento afecta el tratamiento del grano (tierra, piedras), estas impurezas provocan contaminación y destrucción de la muestra a analizar.

Paso 2: Fricción del grano se lleva a cabo en el molino disco de piedra, en el que se raspa el grano y de esta manera obtener muestra perlada (reducción del endospermo para la obtención de harina o semolinas).

Nota: Por diferencia de pesos se obtiene la dureza del endospermo (peso inicial - final).

\section{Hidratación del grano}

El objetivo del acondicionamiento (agregado de agua) o humectación es que las distintas partes del grano adquieran distintas propiedades físicas para facilitar una mejor separación entre ellas.

Con el acondicionamiento se obtiene:

1. Un germen plástico (como la plastilina) que se deforme pero mantenga su tamaño y que no se rompa.

2. Un pericarpio correoso y flexible, que no se pulverice, que se desprenda como tiritas lo más grande posible, para que no contamine la harina.

3. Un endospermo que se pulverice, fácilmente quebradizo.

El proceso de acondicionamiento, del grano es sometido a hidratación en dos tiempos:

1ra Hidratación; con $30 \mathrm{ml}$ de agua por cada 800 gramos de muestra en un tiempo no mayor a 24 horas, para cada variedad.

2da Hidratación; transcurridas las 24 horas de reposo de la muestra con la primera hidratación, la muestra es sometida nuevamente a hidratación hasta alcanzar humedad de $14 \%$ para una mejor fragmentación y obtención de harina fina para las pruebas reológicas.

\section{Molienda en molino experimental Bulher automático}

Para la obtención de harina fina, se somete el grano a dos etapas de molienda

\section{Etapa 1}

Molienda de trigo duro.

\section{Procedimiento}

Paso 1: Pesar la muestra

Paso 2: Introducir la muestra al equipo de molienda para grano duro.

Paso 3: Prender el equipo y esperar tiempo de fragmentación del grano.

Paso 4: Apagar el equipo y sacar la muestra previamente molida.

Etapa 2 Para esta etapa se someten las muestras a un segundo procedimiento mecánico, en el que para obtener una muestra fina, es necesario del molino de rodillo lizo con capacidad de 800 gramos.

Paso 5: Pasar la muestra al equipo de molienda para obtención de muestra fina. 
Paso 6: Prender el equipo y someter la muestra pre-molida hasta obtener harina fina para las pruebas reológicas.

\section{Análisis proximal}

\section{Determinación de Humedad}

Este análisis se determino mediante la cuantificación de materia seca, que permanece en el grano de trigo después de la eliminación del agua, conociéndose como sólidos totales. Este valor analítico es un factor de calidad importante para la conservación y manipulación del grano que en etapa posterior será manipulado para la obtención de harina. Se entiende como harina de trigo, al producto que se obtiene por molienda y tamizado de granos de trigo (BS16, Norteña y Josecha), sanos, limpios, enteros o quebrados así como sin cascara.

Se calcula el contenido de humedad, expresado como peso perdido de la muestra durante el secado siguiendo la fórmula:

\section{\% de humedad: $\frac{P_{i}-P_{f}}{P_{i}} \times 100$}

\section{Determinación de Extracto Etéreo}

En este análisis se obtuvo por el método de extracción de grasa Goldfish, determinando la cantidad de extracto etéreo presente en muestra molida y tamizada de harina de trigo de cada variedad, utilizando la siguiente fórmula se calcula el $\%$ de extracto etéreo.

\section{\% de extracto etéreo: $\frac{P_{f}-P_{0}}{M} \times 100$}

\section{Determinación de Fibra}

Este análisis se basa en el método de digestión ácida y alcalina de la muestra obteniéndose residuo de fibra cruda y sales que con calcinación posterior se determinara la fibra cruda, presente en la muestra analizada. Se calcula el \% con la siguiente expresión.

\% FIBRA: $\frac{\left(P_{\mathrm{s}}-\mathbf{P}_{\mathrm{c}}\right)-\left(\mathbf{P}_{\mathrm{c}}-\mathbf{P}_{\mathrm{cp}}\right)}{M} \times 100$

\section{Determinación de Proteína}

Este análisis se determina por el método Kjeldhal (Cantidad de nitrógeno presente en muestra de trigo molida y tamizada), este es un método indirecto, realmente lo que se determina es la cantidad de nitrógeno presente en la muestra. 
Una vez conocido este al multiplicar la cantidad de nitrógeno obtenida por el factor, se obtiene la cantidad de proteína cruda del producto.

El análisis para determinar el contenido de nitrógeno de la muestra consta de tres fases:

1.- Digestión u oxidación de la materia orgánica.

2.- Destilación de la materia orgánica para desprender el amoniaco que se condensa en una solución ácida.

3.- Titulación de esta solución para determinar el contenido de nitrógeno.

Se calcula el \% de proteína bruta con la siguiente expresión.

\section{$(\mathrm{V} \times \mathrm{f}-\mathrm{V} 1 \times \mathrm{f} 1) 0,014 \times \mathrm{F} \times 100$}

\section{Proteína bruta $\%=\frac{P}{P}$}

\section{Determinación de Cenizas}

Este método gravimétrico está constituido por el residuo inorgánico que se obtiene quedando después de que la materia orgánica se ha calcinado (quemado en su totalidad). Las cenizas obtenidas no tienen la misma composición que la materia mineral presente en el alimento original.

Se determina el \% de ceniza contenida en cada muestra en estudio a través del calculo de porcentaje.

\% de ceniza: $\underline{\left(M_{1}-M_{2}\right) 100}$

$$
\text { M }
$$

\section{Análisis Reológicos}

\section{Método Farinógrafo de Brabender}

Se realiza en equipo Micro-Farinógrafo Brabender Mod-No. 8 110, se midieron las propiedades dinámicas de la masa a través de la resistencia que ésta le opone al amasado mecánico en condiciones controladas, como las siguientes:

1.- Tiempo de homogenización de la muestra.

2.- Volumen de agua necesario para el amasado

3.- Peso de la muestra sometida a amasado.

4.- Tiempo de amasado

\section{Procedimiento}

Paso 1: Pesar la cantidad de acuerdo a la humedad presente en la muestra, analizada en el cuadro estándar para el procedimiento (Micro-Farinógrafo Brabendrer).

Paso 2: Incorporar a la amasadora la harina a analizar en estado seco, solo para homogenizar completamente.

Paso 3: Acomodar el papel regla en la lectura del farinográma partiendo de 0 
por 1 minuto de amasado en seco (para obtener el tiempo óptimo de amasado).

Paso 4: Pasado el minuto de tiempo de amasado en seco, se realiza la descarga de agua destilada sobre la amasadora hasta hidratar por completo la muestra.

Paso 5: Retirar la muestra amasada de las paredes de la amasadora sin tocar sus aspas (Si se tocan se disparará el valor y este resultara erróneo).

Paso 6: Una vez que se llega a las curva en donde se encuentran las 500 unidades Brabender se deja trabajar por 12 minutos.

Del papel regla se obtiene un farinográma del que se extrae la siguiente Información para la interpretación de resultados:

1.- \% de absorción de agua de la harina hasta alcanzar una determinada consistencia (depende de la cantidad y calidad de gluten y la dureza de endospermo, y se relaciona con la cantidad de pan a obtener por kg de harina).

2.- Tiempo de desarrollo de la masa (minutos necesarios para alcanzar la máxima consistencia).

3.- Tiempo de estabilidad o tolerancia al amasado (minutos durante los cuales la masa mantiene la máxima consistencia).

La absorción de agua se define como el porcentaje de agua respecto al peso de harina que es necesario añadir para obtener una masa de consistencia determinada.

Donde:

\section{Absorción de agua $\%=\mathrm{V}+\mathrm{P}-\mathbf{3 0 0} / 3$}

$\mathrm{V}=$ volumen en $\mathrm{ml}$ de agua añadida para obtener una masa con una consistencia máxima de 500 U.B.

$\mathrm{P}=$ peso en $\mathrm{g}$ de harina utilizada, equivalente a $300 \mathrm{~g}$ con el $15 \%$ de humedad.

\section{Alveógrafo de Chopin}

La masa se extiende formando un alveolo, por efecto de la fuerza debida a la presión del aire que se insufla por debajo de una lámina de masa obtenida en condiciones normalizadas. Con este ensayo se imita a gran escala la formación de alveolos en el seno de la masa por el anhídrido carbónico producido por las levaduras durante la fermentación. Las dimensiones y la forma de las curvas obtenidas y el volumen del alveolo en el momento de la rotura son una guía de las características de panificación de la harina.

El procedimiento para la realización de este análisis es el siguiente:

1. Se pesan 60 gramos de harina de cada variedad.

2. Añadir la muestra en la cubeta de amasado.

3. Poner en marcha la amasadora y dejar por 1 minuto la muestra amasando, después adicionar Solución Salina durante 15 segundos. Parar amasadora y homogenizar manualmente la mezcla, con ayuda de una espátula plástica sin tocar las paredes de esta. 
4. Continuar el amasado en la amasadora, durante 8 minutos.

5. Apagar la amasadora y pasar la muestra a las placas de laminado.

6. Lubricar con aceite de vaselina las placas de laminado, posteriormente formar los pastones.

7. Formación de las galletas, para dejar en la cámara de reposo durante 20 minutos y posteriormente pasar al Alveógrafo.

8. Embonar perfectamente el Alveógrafo, insuflar aire y hacer la lectura.

Cálculos:

$\mathrm{W}=132 \times \mathrm{G} 2 \times \mathrm{S} / \mathrm{L}$

Donde:

$\mathrm{L}=$ Longitud de la curva.

$\mathrm{S}=$ Área de la Curva.

$\mathrm{G}=$ Indicé de Inflamiento.

W= Trabajo de Deformación.

\section{Panificación}

Una harina panificable se puede considerar una mezcla de: almidón, electrolitos, agua, gluten. Las propiedades panificadoras dependen de la capacidad de embeber agua del hidrogel, en esto influyen la forma de maduración del trigo y el acondicionamiento de la harina. A la que se añaden levaduras, esto provoca la fermentación de los azucares formándose $\mathrm{CO} 2$ que hace que la masa sea esponjosa. Esta masa esponjosa debe tener otra cualidad: elástica. La elasticidad depende: del número de partículas coloidales del gluten/unidad de masa y de la capacidad de hinchamiento del gluten.

Para llevar a cabo la panificación se realizaron los siguientes pasos:

1. Pesar los ingredientes secos (harina, leche y manteca).

2. Preparar soluciones (levadura y sal-azúcar).

3. Mezclar los ingredientes sólidos y líquidos hasta tener una consistencia suave (que la masa haga hebra y al estirarla sea elástica y flexible).

4. Se pasa la masa al molde de fermentación y se deja reposar en el gabinete de fermentación durante una hora 20 minutos.

5. Se hace el primer fresado (10 movimientos suaves con las palmas de las manos y empujando hacia dentro la masa con los dedos). Se deja reposar 45 minutos en el fermentador.

6. Se hace el segundo fresado (misma operación) y se deja reposar 25 minutos en el fermentador.

7. La masa se moldea mecánicamente (pasa por una prensa dos veces, se enrolla la masa en tres rodillos) y se pasa a moldes dejando reposar la masa 55 minutos en el gabinete de fermentación.

8. Se mete al horno a cocción durante 25 minutos. 
Este procedimiento está basado en el Método 10-10 AACC 1962 de la Asociación Americana de Química del Cereal, el cual se obtuvo en INIFAP.

\section{Evaluación Sensorial}

Es considera simplemente como: el análisis de las propiedades sensoriales, se refiere a la medición y cuantificación de los productos alimenticios o materias primas evaluados por medio de los cinco sentidos. Para obtener los resultados e interpretaciones, la evaluación sensorial se apoya en otras disciplinas como la química, las matemáticas, la psicología y la fisiología entre otras.

\section{Obtención de datos}

1. Al término de su cocción se pesan y se dejan enfriar.

2. Se mide volumen y se guardan para el día siguiente.

3. Se cortan en dos y se evalúan sensorialmente.

Evaluación sensorial (Aptitud panadera)

\begin{tabular}{ll} 
Corteza excelente & 2.0 \\
Volumen mayor que $820 \mathrm{cc}$ & 2.0 \\
Color crema & 2.0 \\
Textura excelente & 2.0 \\
Porosidad excelente & 2.0 \\
Aptitud panadera excelente & 2.0 \\
\cline { 2 - 2 } & 10.0
\end{tabular}

\section{Análisis y discusión de resultados}

En este apartado se expondrán los resultados obtenidos, realizados en el laboratorio de trigo del Instituto Nacional de Investigaciones Forestales, Agrícolas y Pecuarias (INIFAP) y laboratorio de Análisis de los alimentos en el Instituto Tecnológico Superior del Occidente del Estado de Hidalgo (ITSOEH), respectivamente.

Cuadro No.3 Resultados obtenidos de las propiedades físicas y mecánica de las variedades evaluadas.

\begin{tabular}{|l|l|l|}
\hline \multirow{2}{*}{\multicolumn{1}{|c|}{ Variedad }} & \multicolumn{2}{c|}{ Propiedad } \\
\cline { 2 - 3 } & \multicolumn{1}{|c|}{$\begin{array}{c}\text { Física } \\
\text { Peso Hectolítrico }\end{array}$} & \multicolumn{1}{c|}{$\begin{array}{c}\text { Mecánica } \\
\text { Dureza }\end{array}$} \\
\hline Josecha & $73.6 \mathrm{~kg} / \mathrm{hl}$ & 36 \\
\hline Norteña & $75.4 \mathrm{~kg} / \mathrm{hl}$ & 36 \\
\hline Bs16 & $74.4 \mathrm{~kg} / \mathrm{hl}$ & 31 \\
\hline
\end{tabular}


Cuadro No.4 Resultados obtenidos de los Análisis Bromatológicos, realizados en ITSOEH.

\begin{tabular}{|l|l|l|l|}
\hline \multirow{2}{*}{ Análisis } & \multicolumn{2}{c|}{ Variedad } \\
\cline { 2 - 4 } & \multicolumn{1}{|c|}{ Josecha } & \multicolumn{1}{c|}{ Norteña } & \multicolumn{1}{c|}{ BS16 } \\
\hline Humedad \% & 14.15 & 14.27 & 15.61 \\
\hline Extracto Etéreo \% & 2.51 & 2.13 & 2.53 \\
\hline Fibra \% & 1.6 & 1.5 & 1.9 \\
\hline Proteína \% & 17.87 & 17.34 & 19.35 \\
\hline Ceniza \% & 1.8 & 1.76 & 1.8 \\
\hline
\end{tabular}

\section{Alveogramas}

Las expresión de los alveogramas, es calculado por medio de dos curvas obtenidas en el que la expresión máxima es dada por una media de las ordenadas obtenidas en la misma imagen de la hoja de análisis alveografico (véase Anexo III la línea indicada con color rojo, en cada una de las imágenes 2,3 y 4. Las curvas de medida de presión en función del tiempo se caracterizan principalmente por los siguientes parámetros: Pmáx, L, G, W, P/L, Ie.

-Pmáx (mm de H2O): presión correspondiente a la resistencia máxima de la probeta a la deformación. Pmáx depende de la consistencia, de la tenacidad de la masa y del potencial de hidratación de la harina.

-L (mm) y G: extensibilidad de la masa hasta su ruptura.

-G: índice de hinchamiento, es igual a la raíz cuadrada del volumen de aire en $\mathrm{cm} 3$ necesario para el desarrollo de la burbuja.

-W (10-4 J): trabajo de deformación, expresa la fuerza de la harina o su valor panadero.

- P/L: relación de configuración, característica de utilización de la harina.

- Ie (\%) o P200/A más: P200 es la presión medida en el interior de la burbuja después de haber insuflado $200 \mathrm{ml}$ de aire bajo la probeta, está en relación con la resistencia elástica de la masa.

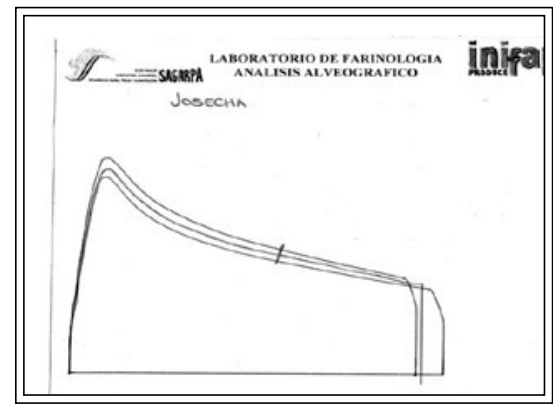

Imagen No.2

Alveograma de la Variedad Josecha.

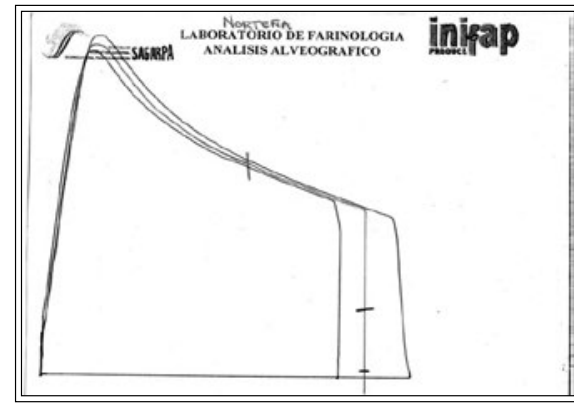

Imagen No.3

Alveograma de la Variedad Norteña. 


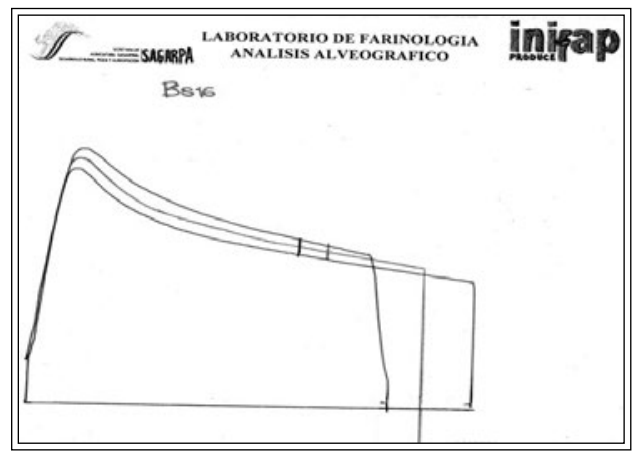

Imagen No. 4 Alveograma de la Variedad BS16.

Las variedades de trigo Josecha y BS16, presentaron características de gluten muy similares entre sí; con valores promedio de: fuerza general 444 x 10-4 Joules, índice $\mathrm{P} / \mathrm{G} 4.0$ e índice $\mathrm{T} / \mathrm{L}$ 0.8, que corresponde a harinas de trigo con gluten fuerte balanceado. La fuerza general indica que pueden ser utilizados en la industria de la panificación mecanizada.

Por otra parte, la variedad Norteña presentó una fuerza general muy elevada 640 x 10-4 Joules, aunque con índices $\mathrm{P} / \mathrm{G}$ y T/L (5.8 y 1.1) que indican la existencia de un gluten balanceado. Dada la gran fuerza del gluten de este trigo, debe considerarse la mezcla de la harina obtenida de la variedad Norteña, con la harina de un trigo de gluten débil balanceado en proporción adecuada, para reducir la fuerza del gluten a niveles adecuados para la industria.

Cuadro No. 5 Resultados obtenidos en Alveógrafo de Chopin, de las tres variedades evaluadas.

\begin{tabular}{|l|l|l|l|}
\hline \multirow{2}{*}{ Alveograma } & \multicolumn{3}{c|}{ Parámetro } \\
\cline { 2 - 4 } & \multicolumn{1}{c|}{ Josecha } & \multicolumn{1}{c|}{ Norteña } & \multicolumn{1}{c|}{ BS16 } \\
\hline Tenacidad $(\mathrm{T}) \mathrm{mm}$ & 87 & 130 & 91 \\
\hline Sobrepresión máxima P= TX1.1 & 95.7 & 143 & 100.1 \\
\hline Extensibilidad (L) mm & 126 & 121 & 119 \\
\hline Expansión o Índice de Hinchamiento (G) & 24.9 & 24.4 & 24.2 \\
\hline Constante de dilatación (C) & 744 & 714.2 & 702.6 \\
\hline Superficie $\left(\mathrm{cm}^{2}\right) \mathrm{S}$ & 66.1 & 98.7 & 70.8 \\
\hline
\end{tabular}

En lo que respecta a los análisis farinográficas, el comportamiento de las 3 variedades estudiadas, fue relativamente diferente en cuanto al porcentaje de absorción de agua, tiempo óptimo de amasado y estabilidad de la masa. 
Josecha: Presentó un menor porcentaje de absorción de agua, seguida por BS16 y Norteña; estos resultados presentan una relación positiva con la fuerza del gluten. Este comportamiento se debe al hecho de que conforme la fuerza del gluten aumenta, la masa de trigo requiere de una mayor cantidad de agua adicionada, a fin de obtener una fuerza estándar de $500 \mathrm{UB}$ en la prueba farinográficas.

El tiempo óptimo de amasado fue diferente para las 3 variedades estudiadas, con diferencias de hasta $3.5 \mathrm{~min}$ entre variedades. A pesar de que las variedades Josecha y BS16 presentaron fuerzas de gluten similares, el tiempo óptimo de amasado fue diferente entre ellas ( 6 y $9.5 \mathrm{~min}$ ), mientras que la variedad Norteña tuvo un tiempo óptimo de amasado de $8 \mathrm{~min}$. Esta variable no presentó relación con la fuerza del gluten determinada en la prueba alveográfica, lo cual indica que independientemente de la fuerza del gluten, la velocidad del arreglo de las moléculas de proteína en el gluten durante el amasado fue diferente en cada una de las variedades estudiadas.

La estabilidad de la masa fue considerablemente mayor ( $>5$ a $6 \mathrm{~min}$ ) para las masas de las variedades Josecha y BS16, condición muy favorable para su uso en la industria mecanizada; en la que las masas son sometidas a un gran esfuerzo mecánico en las amasadoras industriales de gran escala. Por esta razón se demandan harinas cuyas masas resistan el amasado durante un lapso relativamente largo; sin perder fuerza, a fin de que las masas conserven su capacidad de retener gases durante la fermentación, sin perder la estructura del gluten formado durante el amasado.

Al respecto, las masas que conservan su estabilidad por más de 12 min se consideran de alta calidad industrial. La masa que presentó la mayor tolerancia al mezclado fue la de la variedad BS16, pues fue la que mostró una menor reducción de la fuerza de la masa, al cabo de un mezclado estandarizado por un tiempo determinado. Este resultado es consistente con el mayor tiempo óptimo de mezclado observado para la misma variedad.

A pesar de este comportamiento, las 3 variedades estudiadas presentaron buena tolerancia al mezclado.

Cuadro No.6 Resultados de Farinógrafo Brabender, de las tres variedades evaluadas.

\begin{tabular}{|l|l|l|l|}
\hline \multirow{2}{*}{ Farinográma } & \multicolumn{3}{c|}{ Parámetro } \\
\cline { 2 - 4 } & \multicolumn{1}{|c|}{ Josecha } & \multicolumn{1}{c|}{ Norteña } & \multicolumn{1}{c|}{ BS16 } \\
\hline Absorción de Agua \% & 57.5 & 59.1 & 58.6 \\
\hline Tiempo óptimo de amasado min & 6 & 8 & 9.5 \\
\hline Estabilidad de la masa min & 14 & 9 & 15 \\
\hline Índice de Tolerancia UB & 80 & 80 UB & 70 UB \\
\hline
\end{tabular}


Cuadro No.7 Resultados obtenidos de la Evaluación Sensorial, de las variedades evaluadas

\begin{tabular}{|l|l|l|l|l|l|l|l|}
\hline \multirow{2}{*}{ Variedad } & \multicolumn{7}{|c|}{ Evaluación Sensorial (Aptitud panadera) } \\
\cline { 2 - 8 } & $\begin{array}{c}\text { Corteza } \\
\text { excelente }\end{array}$ & $\begin{array}{c}\text { Volumen } \\
\text { mayor que } \\
820 \mathrm{cc}\end{array}$ & $\begin{array}{c}\text { Color } \\
\text { crema }\end{array}$ & $\begin{array}{c}\text { Textura } \\
\text { excelente }\end{array}$ & $\begin{array}{l}\text { Porosidad } \\
\text { excelente }\end{array}$ & $\begin{array}{c}\text { Aptitud } \\
\text { panadera } \\
\text { excelente }\end{array}$ & Total \\
\hline Josecha & 1.5 & 1.5 & 1.5 & 1.5 & 1.5 & 1.5 & 9 \\
\hline Norteña & 1 & 1 & 1 & 1 & 1 & 1 & 6 \\
\hline BS16 & 2 & 2 & 2 & 2 & 2 & 2 & 10 \\
\hline
\end{tabular}

\section{Conclusiones}

Se realizó la evaluación agroindustrial de 3 variedades pre comerciales de trigo (BS16, Norteña y Josecha) cultivadas en la Región de Cinta Larga - Mixquiahuala, Hgo. En donde la variedad Norteña presento el mayor rendimiento con 3.770 toneladas por hectárea cultivada.

En el análisis del manejo del grano se obtuvo que la variedad JOSECHA presento un porcentaje de grano sano del $89 \%$.

Norteña por su parte presento el mayor peso hectolítrico $(75.4 \mathrm{~kg} / \mathrm{hl})$ y la variedad BS16 el menor índice de perlado, es decir la mayor dureza (31).

Uno de los parámetros más importantes en el análisis bromatológico es la proteína presente en el grano de trigo, la variedad que presentó el mayor porcentaje de este componente fue la variedad BS16 con $19.35 \%$.

En los análisis reológicos realizados a las tres variedades de trigo se concluye que la variedad NORTEÑA presentó una masa con gluten considerablemente más fuerte y tenaz que las variedades JOSECHA y BS16; por lo que estas dos últimas variedades presentan mejor calidad para su uso en panificación. Por lo anterior, se sugiere para que la harina de la variedad NORTEÑA pueda ser utilizada adecuadamente en la panificación, se mezcle con harina de un trigo de gluten débil extensible, para obtener un mejor comportamiento panadero. La absorción de agua que la harina de trigo requiere para el mezclado y formación de la masa la variedad BS16 arrojo el mejor porcentaje que fue de $58.6 \%$, por lo que su rendimiento panadero en peso será mayor.

En la evaluación sensorial la variedad que obtuvo el mayor puntaje de 10; que corresponde a una calificación de excelente fue la variedad BS16 con las mejores características sensoriales.

Finalmente se determina que la variedad BS16 es la más apta para la industrialización, ya que los parámetros óptimos fueron obtenidos en esta variedad. 


\section{Referencias Bibliográficas}

Desrosier, 1999

David. Dendy Cereales y productos derivados (Química y Tecnología),. Phd, Bodgan J.Dobraszczyk, Phd Editorial Acribia, S.A. Zaragoza (España).

Composición y Análisis de Alimentos, Pearson. Ronald S. Kirk, Ronald Sawyer., Harold Egan. Séptima Reimpresión, México 2005.

Complementos de Bromatología, Departamento de Química Orgánica - Área Bromatología. Facultad de Ciencias Exactas y Naturales. Universidad de Buenos Aires.

Industrias de Cereales y Derivados. María de Jesús Callejo González. Primera Edición Año 2002, AMV Ediciones. Mundi Prensa.

Manual de Prácticas de Bromatología, Universidad Nacional Autónoma de México. Escuela Nacional de Estudios Profesionales. Zaragoza.

Tecnología de los Cereales. Introducción para Estudiantes de Ciencias de los Alimentos y Agricultura. Editorial Acribia, S.A. N.L. Kent. Impreso en España.

NMX-F-007-1982. Alimento Para Humanos. Harina De Trigo. Foods For Humans. Wheat Flour. Normas Mexicanas. Dirección General De Normas.

NMX-F-089-S-1978. Determinación De Extracto Etéreo (Método Soxhlet) En Alimentos. Foodstuff-Determination Of Ether Extract (Soxhlet). Normas Mexicanas. Dirección General De Normas.

NMX-F-066-S-1978. Determinación De Cenizas En Alimentos. Foodstuff Determination Of Ashes. Normas Mexicanas. Dirección General De Normas.

NMX-F-068-S-1980. Alimentos. Determinación De Proteínas. Foods. Determination Of Proteins. Normas Mexicanas. Dirección General De Normas. 